\title{
Polysaccharides in Pharmacy: Current Applications and Future Concepts
}

\author{
G. Franz \\ Department of Pharmacy, University of Regensburg, D-8400 Regensburg, Federal Republic of Germany
}

Received: February 6, 1989

\section{Abstract}

Polysaccharides constitute a structurally diverse class of biological macromolecules with a wide range of physicochemical properties, which are the basis for the different applications in the broad field of pharmacy and medicine. Besides the classical applications of these biopolymers in industry and pharmaceutical practice, the relatively new field of the physiologically active polymers will be discussed. Some examples will be given for the socalled immune-modulating antitumor polysaccharides which have been shown to be prominent candidates for an adjuvant tumor therapy.

\section{Introduction}

Several hundred natural polysaccharides are currently known and provide one of the richest and oldest reservoirs of structurally and functionally diverse biopolymers. Polysaccharides have played an essential role of life in general and, furthermore, constitute important members of the family of industrial water-soluble polymers (1).

Terminology in the field of plant polysaccharides such as gums and mucilages which was vague though colourful is currently yielding to more precise designations. A gum has the subjective attribute of being gummy, a mucilage of being mucilaginous. The terms gum and mucilage have often been used interchangeably, although the pharmacist generally considers a mucilage as being a solution of a gum.

All commercial gums are of plant origin, so that there is really no gain in speaking of "plant" gums. There is, however, a necessary distinction in the appelation of "watersoluble" gums, to distinguish them from similar appearing tree exduates, which are insoluble in water but soluble in organic solvents.

There is general agreement that water-soluble gums are polymer materials which are more or less soluble in water to form a viscous solution, a geletinous paste, or a jelly depending on the concentration employed.
Further use of the subjective terms gums and mucilage as group designations can hardly be justified, since application of the terms is usually arbitrarily restrictive. A more generally inclusive and descriptive name for this group of substances would be "polysaccharide hydrocolloids".

Polysaccharide hydrocolloids are abundant in na.ture, present in many plant sources, but only a restricted number is of commercial importance. (Table 1). The economic justification for the production and utilization of each polysaccharide is complex and varies from one geographical area to the next. For medical and pharmaceutical purpose many uses have been established and many new ones are constantly being discovered (2).

Table 1 Herbal drugs containing polysaccharide hydrococolloids actually used in the Federal Republic of Germany.

\begin{tabular}{ll}
$\begin{array}{l}\text { High content of } \\
\text { Polysaccharide }\end{array}$ & $\begin{array}{l}\text { Low content of } \\
\text { Pydrocolloids }\end{array}$ \\
\hline Althaea officinalis & Hydrocolloids \\
Plantago psyllium & Tilia cordata \\
Plantago indica & Tilia platyphyllos \\
Plantago ovata & Verbascum $\mathrm{sp}$. \\
Linum usitatissimum & Tussilago farfara \\
Lichen islandicus & Malva silvestris \\
Symphitum officinalis & Plantagolanceolata \\
\hline
\end{tabular}

The polysaccharides actually used for pharmaceutical purposes can be devided in two groups: the natural and the modified natural hydrocolloids (Table 2).

The susceptibility of polysaccharides to a broad range of chemical and enzymatic transformations provides additional opportunities for the extension of their utility. Countless methods are being used and developed for polysaccharide modifications resulting in the development of new useful derivatives (3).

The importance of various polysaccharide hydrocolloids for pharmaceutical purposes has a long historical background and increased considerably during the last decades (Table 3). This is not only the case for polysaccharides used as excipients essential for specific drug formulations, but also for a series of carbohydrate polymers which have proven to be most useful as physiologically active drugs (4). Many in- 
Table 2 Classification of natural and semisynthetic modified polysaccharide hydrocolloids.

\section{Algal (seaweed) \\ polysaccharides \\ A. Red seaweed extracts Agar, Carrageenan \\ B. Brown seaweed extracts Alginic acid, Laminarin}

\section{Plant polysaccharides \\ A. Plant exudates Gum arabic, Gum ghatt Gum karaya, Gum tragacanth \\ B. Plant seed polysaccharides Guar gum, Locust bean gum \\ C. Plant extracts Pectin, Larch gum}

\section{Cellulose derivatives}

Carboxymethylcellulose Hydroxyethylcellulose Hydroxypropylcellulose Methylcellulose Microcrystalline cellulose

IV. Microbial polysaccharides Xanthan, Dextran

Scleroglucan

Curdlan, Pullulan

Zanflo 10

Baker's yeast glycans Schizophyllan, Lentinan Krestin
Table 3 Functional properties of polysaccharide hydrocolloids.

\section{Function}

Stabilizer, suspending agent

Thickener, film-forming agent

Water retention agent, coagulant

Colloid, lubricant or friction reducer

teresting areas have been opened in the past which include their role in drug delivery, in wound treatment, in cancer therapy, and the diagnosis, prevention, and treatment of bacterial and viral deseases.

Compendial standards and governmental regulations require that even the natural polysaccharides as well as their derivatives meet the strict standards of identity, potency, and purity, in order to define the necessity of drug quality (Table 4). Some polysaccharides may undergo chemical changes with time, resulting in a loss of biological and therapeutical activity (5).

Table 4 Criteria for the pharmaceutical utilization of polysaccharides.

\begin{tabular}{ll}
\hline Polysaccharides must have: & Polysaccharides must not: \\
\hline - high purity & - inactivate enzymes \\
- chemical, physical, and & - cause cancer \\
mechanical properties & - produce toxic or allergic \\
for the proposed & reactions \\
$\quad$ functions & - deplete electrolytes \\
- high stability & \\
- easy fabricability & \\
\hline
\end{tabular}

Recent developments of analytical techniques are the basis for the guide-lines for an efficient control of polysaccharide hydrocolloid quality even if they are present in complex mixtures or drug formulations (6). The following criteria should be elucidated:

1. Determination of the molecular composition of a carbohydrate polymer in question; i.e. sugar analysis by GLC.
2. Determination of the relative molecular mass of the polysaccharide in question by GPC and other methods, further the homogeneity or heterogeneity of a polysaccharide fraction.

3. Rheological properties of the polymer.

\section{General Properties of Polysaccharides}

Solubility is a very important criterium for the different uses of these polymers in pharmacy. Polysaccharides containing carboxyl groups, such as pectins, alginates, and carboxymethylcellulose, are insoluble at low $\mathrm{pH}$ values. They will be precipitated when the $\mathrm{pH}$ is lowered below 3 .

Pectins and alginates are generally insoluble in solutions containing divalent ions. An exception is the solubility of alginates in solutions containing magnesium ions. Solubilization of a polysaccharide can sometimes be achieved if a chelating agent is included in the formulation. In general, the maximal concentration of a water-soluble polysaccharide is limited by the viscosity. As a consequence, higher concentrations can be obtained with low molecular mass fractions.

Polysaccharides in solution will depolymerize when the solution is heated and this will cause a drop in viscosity. The intensity of this phenomenon for any given time/temperature régime depends primarily on the polysaccharide structure and $\mathrm{pH}$. Almost all polysaccharides depolymerize in solution, more rapidly in acid than in neutral or alkaline media. An exception is xanthan gum which is extremely stable under all conditions, and further pectin, which is more stable in acid than in neutral media.

\section{Medical use of Polysaccharide Hydrocolloids}

The compendium of all the formulations actually produced in the Federal Republic of Germany, the "Rote Liste 1988", lists polysaccharide hydrocolloids in the following main sections: Antitussiva, Antiphlogistica, Dermatica, Dietetics, Laxantia, Stomachica, Immune-stimulators.

It is, however, important to note that in almost all commercial products the polysaccharide hydrocolloids never occur alone but are part of a more or less complex formulation with other plant constituents or chemical compounds.

The use of polysaccharide hydrocolloids in antitussive formulations such as cough drops, cough syrup, cough lozenge is well documented. As a consequence of inflammatory events of the mucous membranes in the respiratory tract, the naturally occurring mucin layer is disturbed in its function (7). After a medication with polysaccharide hydrocolloids, the mucin effect can be restored by forming a protective layer in order to desensitize the irritated peripheroceptors. Local inflammations are diminished by this effect, and as a consequence, the dry cough will be reduced. By using a polysaccharide hydrocolloid containing herbs for the preparation of an infusion, in addition, the thermogenic-hyperemic effect will be helpful.

A positive physiological effect of some polysaccharide hydrocolloids is known to occur in the intestinal system. However, it is important that these polysaccharides are not degraded by microbial enzymes, in order to guarantee the 
physico/chemical, i.e. film-forming, properties. Intestinal irritations will be reduced and, further, the polysaccharide hydrocolloid film can function as a resorption barrier for toxins.

Polysaccharide hydrocolloids are an almost ideal treatment for the common constipation. Lin seed, quince seed, or psyllium (Plantago) are the classical herbal drugs which are used alone or in combination with other laxative systems. For the desired effect, both the rheological properties and the increase in volume are useful. It is generally accepted and of great importance that all these high-fiber and high-polysaccharide hydrocolloid preparations have to be used with the adequate amount of liquid, otherwise a reverse effect i.e. colon obstruction will be possible (8).

Topical application of polysaccharide hydrocolloids may have an indirect or direct effect on the area of application. Semisolid systems fulfill a special topical need by being able to cling to the surface of application. Such systems are plastic in behavior, which allows semisolids to be mechanically spread as an immobile film. For the production of lipidfree ointments, pastes, and creams several gel-forming polysaccharides are being used. As an emulsifier they can provide a three-dimensional matrix which determines the extent and the quality of the gel-like micellar structure.

For external application, the polymer is usually present in low concentrations ranging from $0.5-2 \%$. Some systems are clear, others are turbid when the polymer is not fully dissolved or is forming aggregates. The natural polymers frequently used for the preparation of pharmaceutical gels include tragacanth, pectin, carrageenan, agar, alginic acid, as well as semisynthetic polysaccharides such as methylcellulose, hydroxymethylcellulose, and carboxymethylcellulose.

For a therapeutical use of polysaccharide-containing preparations as cataplasms, as for example fenugreekseed, linseed or Symphitum root extracts, most likely the heat/ water capacity of these local applications is essential by which the peripheral blood flow is stimulated with all the relevant consequences.

Ointments containing polysaccharide hydrocolloids, such as the Symphitum fructans, show a waterbinding, a moistening, and an anti-inflammatory effect (9). But it is not clear whether this is due to the local hydration or a direct effect of the biopolymers on the cellular functions.

Branched glucans of Dictyophora sp. have been shown to exhibit anti-inflammatory effects on both carrageenan-induced edema and scalded edematous hyperalgesia in rat's hind paws (10). Similar results were obtained with glucans from the fruit bodies of Auricularia species (11).

\section{New Areas for Polysaccharide Application in Medicine}

It has been known for some time that, in animal experiments, a high polysaccharide diet reduces both the blood level of cholesterol and of glucose. This was shown with undigestible gelling polysaccharides such as pectins, tragacanth, guar, and others (12).
Clinical experiments gave comparable results in humans. The best effects were obtainted with different galactomannan preparations such as, for example, locust bean (guar) (12). However, in order to reach the necessary reduction of unphysiological blood glucose values, relatively high amounts of polysaccharide hydrocolloids have to be swallowed with an appropriate amount of liquid. These quantities may have side effects such as meteorism, which is due to a microbial degradation of galactomannans.

These glucose- and cholesterol-lowering effects are due to the formation of an intestinal resorption barrier and, in part, to an amylase inactivation.

Some recent results with extracellular glucans of bacterial origin had the similar effects as galactomannans. An Acetobacter polysaccharide with a very high viscosity was shown to depress the postprandial rise of the glucose blood concentration. It is speculated that the effect is in close correlation with the viscosity of the polysaccharide solutions (13).

In recent experiments whole series of polysaccharide hydrocolloids were tested for their direct hypoglycemic activity $(14,15)$. In this case the blood glucose lowering is not related to the film-forming effect, i.e. formation of a diffusion barrier. In these experiments the polysaccharide hydrocolloids were given $i$.p. to male mice. Blood was drawn from the orbital sinus after treatment and the glucose level in the plasma was determined and expressed as the relative glucose level. The authors could demonstrate a dose dependent effect of blood glucose lowering up to $50 \%$. Even a structure/activity relationship was proposed showing clearly that a polysaccharide unit composed of galacturonic acid, glucuronic acid, and rhamnose is essential for this effect $(16,17)$. However, physiological explanations for this phenomenon were not given, and it is further uncertain if the neccessary high polysaccharide dosis $(100 \mathrm{mg} /$ $\mathrm{kg}$ ) can be tolerated by humans.

\section{Polysaccharides as “Biological Response Modifiers" (BMR)}

BRM's have been defined as those agents or approaches that modify the host's biological response by a stimulation of the immune system, which may result in various therapeutic effects (17). Some of these agents are called immuno-modulators. The actually used BRM's include synthetic and biological agents with a series of completely different chemical structures.

Several herbal drugs are actually considered as being potent in this area (Table 5). Besides low molecular mass compounds, polysaccharides were postulated to have an unspecific immune-modulation effect as has been documented with a variety of these polymers (18). The best documented herbal drug in this field is Echinacea sp. (19). Extracts of this plant are included in several immune-stimulating formulations commonly used to increase the resistance against viral or bacterial infections (20).

The effectiveness of these preparations is still a matter of controversy since it is not known if oral applications of polymeric immuno-stimulants, such as polysaccharides, are taken up by the intestinal system in an undergraded form. 
Table 5 Herbal drugs containing immune-stimulating polysaccharides (20).

\begin{tabular}{ll}
\hline Achyrocline satureioides & Echinacea angustifolia \\
Angelica acutiloba & Echinacea purpurea \\
Arnica montana & Eleutherococcus senticosus \\
Astragalus gummifera & Eupatorium cannabinum \\
Astragalusmongholicus & Eupatorium perfoliatum \\
Avena sativa & Oryza sativa \\
Bambusa vulgaris & Sabal serrulata \\
Baptisia tinctoria & Saccharum officinarum \\
Calendula officinalis & Triticum sativum \\
Carthamus tinctorius & Viscum album \\
Chamomilla recutita & \\
\hline
\end{tabular}

\section{Antitumor Polysaccharides}

Within the field of pharmacologically active polymers, the area of immune-stimulating polysaccharides is a rather new and attractive field of tumor therapy (21). The interest in polysaccharides as antitumor substances came from the dissatisfaction with current cancer chemotherapy. Countless numbers of chemical compounds have been tested as cytostatic agents with possibly a high specificity for the cancer cell. But unfortunately in many cases, this specificity is rather low, and therefore many chemotherapeutic antitumor substances have considerable side effects. Extensive studies have been carried out on nontoxic antitumor substances and most interestingly, some polysaccharides were shown to be most useful (22).

Mainly fungal polysaccharides have been reported to be active against a series of allogeneic and syngeneic tumors (23) (Table 6). These antitumor polysaccharides, in most cases, were shown to be glucans with different types of glycosidic linkages. Some structural features were obvious prerequisites; i.e. $\beta$-1,3-linkages in the main chain of the glucan and further $\beta-1,6$-branch points. It is interesting to note that besides these very active glucans, several fructans were shown to exhibit pronounced activity against a series of different tumor types (24).

Table 6 Polysaccharides with an antitumor effect.

\begin{tabular}{|c|c|c|}
\hline Substances & Sources & Chemical structure \\
\hline $\begin{array}{l}\text { Lentinan } \\
\text { Schizophyllan } \\
\text { Pachymaran } \\
\text { Krestin (PS-K) }\end{array}$ & $\begin{array}{l}\text { Basidiomycetes: } \\
\text { L. edodes } \\
\text { S. commune } \\
\text { P. cocos } \\
\text { C. versicolor }\end{array}$ & $\begin{array}{l}\beta-1,6 ; \beta-1,3 \text {-glucan } \\
\beta-1,6 ; \beta-1,3 \text {-glucan } \\
\beta-1,3 \text {-linear glucan } \\
\beta-1,4 ; \beta-1,3 ; \beta-1,6 \text {-glucan } \\
\quad \text {-protein }(25-38 \%) \text { complex }\end{array}$ \\
\hline$A_{1}$ Glucan & $\begin{array}{l}\text { Oomycetes: } \\
\text { Phytophthora } \\
\text { parasitica }\end{array}$ & $\beta-1,6 ; \beta-1,3$-glucan \\
\hline $\begin{array}{l}\text { Glucan (particulate } \\
\text { and soluble) } \\
\text { Mannozym } \\
\text { Scleroglucan }\end{array}$ & $\begin{array}{l}\text { Yeast: } \\
\text { S. cerevisiae } \\
\text { S. cerevisiae } \\
\text { S. glucanicum }\end{array}$ & $\begin{array}{l}\beta \text {-1,3-glucan } \\
\text { glucomannan } \\
\beta-1,6 ; \beta-1,3 \text {-glucan }\end{array}$ \\
\hline $\begin{array}{l}\text { Curdlan } \\
\text { Levan }\end{array}$ & $\begin{array}{l}\text { Bacteria: } \\
\text { A. faecalis } \\
\text { A. levanicum }\end{array}$ & $\begin{array}{l}\beta-1,3 \text {-glucan } \\
\beta-2,6 \text {-fructan }\end{array}$ \\
\hline Fructan & $\begin{array}{l}\text { Higher Plants: } \\
\text { Solidago virgaurea }\end{array}$ & $\beta-1,2$-fructan \\
\hline
\end{tabular}

At the moment there are three polysaccharides in clinical use: Schizophyllan, Lentinan and Krestin. The fungal polysaccharides originating from Schizophyllum commune and Lentinus edodes were shown to posess the basic structure of a $\beta$-1,3-glucan with $\beta$-1,6-glucopyranosidic branches for every third glucose unit. Krestin, which is isolated from Coriolus versicolor, is a $\beta$ - 1,4 -glucan with $\beta$-1,6-glucopyranosidic side chains for every fourth glucose unit, containing some peptide residues. The clinical usefulness of these polysaccharides has been demonstrated with patients having head and neck cancer, lung cancer, gastric cancer, and cervical cancer. These polymers were either used alone or in combination with chemotherapy and/or radiotherapy, but in most cases after the surgical removal of the primary tumor $(22,25)$.

As a result, it was observed that the immunological parameters of the patientimproved, and the survival periods were considerably prolonged. Side effects during the polysaccharide treatment were rarely observed, mostly occurring as local irritations at the injection site, seldom as fever or vomitting. In general, intramuscular injections of up to seven times a week with a single dose, ranging from 5 to $50 \mathrm{mg}$ of these polysaccharides, were shown to be tolerable by cancer patients $(26,27)$.

For the search of new antitumor polysaccharides, the allogeneic tumor Sarcoma 180 is generally used as the basic screening model. This tumor can be easily transplanted into female CD1-mice. In a typical experiment $5 \times 10^{6}$ cells are subcutaneously injected at day 0 of the experiment (28). The following tumor growth is very rapid during the experimental period of 30 days. An increase of the tumor diameter can be measured easily without killing the mice.

The polysaccharides, which are used for the tumor treatment, are usually dissolved in a saline medium. Control animals are treated with the saline medium only. The solutions are interperitoneally injected at $24 \mathrm{~h}$ intervals for 10 consecutive days. The rate of inhibition is calculated by comparing the average tumor weight of the polysaccharide treated animals with the average tumor weight of the control group. The regression rate is determined as the number of completely cancer-free animals in relation to the total number of animals used for the experiment (29). In some cases it can be seen that under polysaccharide treatment the implanted tumor is in a steady regression, or after 30 days under optimal conditions, the animal is devoid of tumor cells.

Several polysaccharides originating from higher plants were shown to have a relatively moderate effect on the growth of the Sarcoma 180. However, a certain structure/activity relationship could be established (30).

Convincing results were obtained with a branched glucan isolated from the cell walls of the fungus Phytophthora parasitica. This polymer has some structural resemblance with Schizophyllan, i.e. a $\beta$-1,3-backbone and 1,6branch points. In addition, $\beta$-1,3-linkages are present in the side chains (31). This polysaccharide proved to be an very potent immune-modulating agent with an antitumor activity comparable or even better than that of Schizophyllan (32). 
With this Phytophthora glucan $\mathrm{A}_{1}$ a close activity/dose relationship and a relative molecular mass dependent activity could be demonstrated. The glucan with the highest degree of polymerization (DP) showed optimum activity (32).

In all these experiments it is typical that tumor growth is not influenced during the first 10 days of polysaccharide treatment. After this initial experimental period, tumor growth is negatively influenced. This is an indication for the indirect, non-cytotoxic effect which is due to the immunestimulating capacity of the carbohydrate polymer. This typical BRM effect was substantiated by the in vitro Sarcoma 180 Stamm-cell assay, where the polysaccharides had no antineoplastic activity (28).

The result of an animal pretreatment prior to tumor implantation with the Phytophthora polysaccharide $\mathrm{A}_{1}$ is similar to the direct polysaccharide effect. Here again, an initial tumor growth can be observed with a following decline of the tumor diameter. This pretreatment effect again is an indication for the indirect effect of the Phytophthora polysaccharide.

It has been postulated that this antitumor effect is in part due to macrophage activation (33). In order to prove this hypothesis, mice were treated with trypan-blue, a specific macrophage inhibitor. During the treatment with this dye, the activity of the Phytophthora glucan was slightly diminished, compared to the animals which were treated in the normal way without trypan-blue. The substance itself had no influence on the tumor growth. From these experiments it can be concluded that a macrophage activation is only in part responsible for the antitumor effect of the polysaccharide in question (33).

Besides the allogeneic tumor Sarcoma 180, syngeneic tumor models such as Lewis lung cancer, DBAMC.CS-1 Fibrosarcoma, EL-4-Lymphoma and others have to be tested, and further, induced primary tumors should as well respond to the immune-modulating substances. Furthermore, the immune-stimulating activities have to be demonstrated for the individual immune-competent cellular systems: $i$.e. the $T$ cell participation, macrophage participation, complement system activation etc. Finally, a close structure-activity relationship can be established by chemically modifying the polysaccharide structures (34).

\section{Acknowledgements}

We are grateful for financial support by the "Deutsche Krebshilfe" and the "Fonds der Chemischen Industrie". Fruitful discussions with Dr. W. Blaschek, Dr. J. Kraus and the encouraging help of Prof. Dr. H. Schönenberger are acknowledged.

\section{References}

1 Franz, G. (1985) in: Polysaccharide: Eigenschaften und Nutzung, (Burchard, W., ed.), pp. 1-30, Springer Verlag, Berlin, Heidelberg, New York.

2 Franz, G. (1985) ibid., pp. 126-141.

${ }^{3}$ Balser, K. (1985) ibid., pp. 84-110.

${ }^{4}$ Franz, G. (1985) Adv. Polymer Sci. 1-30.

5 Madaus, A. (1989) Dissertation, University of Regensburg.

${ }^{6}$ Franz, G. (1987) Farm. Tijdschrift voor Belgie 4, 301-311.

7 Kartnig, T. (1986) Österr. Apoth. Ztg. 40, 941-945.

8 Franz, G, (1984) Euromed 2, 69-73.

9 Koehler, H., Franz, G. (1987) Z. Phytotherapie 8, 166-168.

${ }^{10}$ Hasa, Ch., Kiko, T., Tanaka, Y., Ukai, S. (1982) Carbohydr. Res. 110, $77-85$.

11 Kiko, T., Sakai, M., Ukai, S. (1985) Carbohydr. Res. 142, 344-350.

12 Leeds, A. R. (1982) in: Dietary Fiber in Health and Desease, (Vahonny, G., Kritchevsky, D., eds.), Plenum Publishing Corp., New York, pp. 323-340.

13 Gatti, E., Cattezano, G., Camisaca, E., Torii, A. (1984) Ann. Nutr. Metab. 28, 1-7.

14 Modiziumi, S., Hata, H., Ebihara, K. (1986) Agric. Biol. Chem. 50, $511-525$

15 Tomoda, M., Shimada, K., Konno, Ch. (1986) Carbohydr. Res. 147, $160-163$

${ }^{16}$ Tomoda, M., Shimada, K., Konno, Ch., Hikino, W., (1984) Planta Med. 365-367.

17 Tomoda, M., Shimizu, N., Oshima, Y., Takahashi, M. (1987) Planta Med. 53, 8.

${ }^{18}$ Herberman, R. B. (1987) Clin. Physiol. Biochem. 5, 238-248.

19 Bauer, R., Wagner, H. (1988) Z. Phytotherapie 9, 151-160.

20 Wagner, H. (1983) Naturheilpraxis 3, 256-270.

21 Whistler, R. L., Bushway, A. A., Singh, P. P. (1976) Advances in Carbohydrate Chemistry and Biochemistry, Vol. 32, 517-535.

22 Furue, H. (1987) Drugs of Today 23, 335-346.

${ }^{23}$ Chihara, G., Hamuro, J., Maeda, Y., Shiio, T., Sunja, T. (1987) Immunbiology of Cancer and Aids, (Niebugs, N. E., ed,), A. R. Liss. Inc. New York, pp. 423-438.

${ }^{24}$ Kraus, J., Schneider, M., Franz, G. (1986) Dtsch. Apoth. Ztg. 126, 2045-2049.

${ }^{25}$ Chihara, G. (1984) EOS Ric. Immunol. Immunfarmacol 4, 85-115.

${ }^{26}$ Chihara, G., Hamuro, J., Maeda, Y. Y., Shiio, R. (1987) Cancer Detection and Prevention 1, 423-443.

27 Matsuo, T., Arika, M., Mitani, M., Komatsu, N. (1982) Drug Res. 32 , 647-657.

28 Franz, G., Kraus, J. (1988) Z. Phytotherapie 8, 114-118.

29 Franz, G., Kraus, J. (1988) Therapeuticon 1, 291-296.

30 Kraus, J., Franz, G. (1989) unpublished data.

31 Bruneteau, M., Fabre, J., Perret, J., Michel, G., Ricci, P., Joseleau, J. P., Kraus, J., Blaschek, W., Franz, G. (1988) Carbohydr. Res. 175, $137-143$

${ }^{32}$ Kraus', J. (1987) Dissertation, University of Regensburg.

${ }^{33}$ Kraus, J., Blaschek, W., Franz, G. (1988) Arch. Pharm. 321, 630.

${ }^{34}$ Hensel, A., Kraus, J., Franz, G. (1988) Dtsch. Apoth. Ztg. 128, 13051309. 